\title{
Buena administración, transparencia y eficiencia: evidencia de los municipios de Colombia
}

\author{
David A. Ortiz Escobar ${ }^{1}$ \\ Sergio Ordóñez Beltrán ${ }^{2}$
}

\section{RESUMEN}

El artículo argumenta que la transparencia es un elemento constitutivo de la buena administración, mientras que la eficiencia debe ser entendida como el resultado de la buena administración en general, y de la transparencia en particular. Se analiza la relación entre transparencia y eficiencia de los gobiernos municipales de Colombia a través del Índice de Gobierno Abierto (IGA) y del componente de eficiencia del Índice de Desempeño Integral (IDI) del Departamento Nacional de Planeación (DNP). Encontramos una asociación importante, estadísticamente significativa, entre el cumplimiento normativo de las normas sobre manejo y difusión de información, y la eficiencia de las administraciones municipales en la provisión de ciertos bienes y servicios.

1 Docente e investigador de la Facultad de Economía de la Universidad Externado de Colombia, Bogotá, Colombia. PhD en Economía de la Universidad George Mason, Fairfax, Virginia, Estados Unidos. Correo-e: david.ortiz@uexternado.edu.co. Enlace ORCID: https:// orcid.org/0000-0002-0433-517x.

2 Estudiante de octavo semestre de Economía, Universidad Externado de Colombia, Bogotá, Colombia, y Aix-Marseille Université, Marsella, Francia. Correo-e: sergio.ordonez01@ est.uexternado.edu.co. Enlace ORCID: https://orcid.org/0000-0003-4500-1835. Fecha de recepción: 15 de agosto de 2018. Fecha de modificación: 30 de agosto de 2018. Fecha de aceptación: 24 de septiembre de 2018. Para citar el artículo: Ortiz Escobar, David A., Ordóñez Beltrán, Sergio. "Buena administración, transparencia y eficiencia: evidencia de los municipios de Colombia", Revista digital de Derecho Administrativo, Universidad Externado de Colombia, n. ${ }^{\circ} 21,2019$, pp. 179-199. DOI: https://doi.org/10.18601/21452946.n21.09 
Palabras clave: buena administración, transparencia, gobierno abierto, eficiencia, buen gobierno.

\title{
Good Administration, Transparency and Efficiency: Evidence from Colombian Municipalities
}

\begin{abstract}
This paper argues that transparency is a main element of good administration, while efficiency must be understood as the result of good administration in general and of transparency in particular. The research explores the relationship between transparency and efficiency in Colombian municipalities by comparing the Open Government Index (IGA) with the efficiency component of the National Planning Agency's Integral Performance Index (IDI). It finds an important and statistically significant association between the fulfillment of legal requirements regarding information and greater levels of efficiency of local provision of certain goods and services.

Keywords: Good administration, Transparency, Open Government, Efficiency, Good Governance.
\end{abstract}

\section{INTRODUCCIÓN}

Entre 1980 y 1990, la mayoría de los países de América Latina y el Caribe emprendieron un proceso de reforma del Estado, entre otras cosas, enfocado en la descentralización política y fiscal, que permitió el florecimiento del poder local bajo el postulado de que la profundización de la democracia pasaba por la participación de los ciudadanos, la apertura de las instituciones públicas y el establecimiento de mecanismos de rendición de cuentas. Además, las reformas se basaron en la idea de que la atención de las demandas de los ciudadanos por parte del Estado debía hacerse a un nivel más cercano al ciudadano: en el municipio o poder local ${ }^{3}$.

3 Para un análisis del proceso de descentralización en América Latina, véase Tulia G. FaLleti, Decentralization and subnational politics in Latin America, Cambridge: Cambridge University Press, 2010; Diego E. Pinilla-RodrígueZ, Juan de Dios Jiménez Aguilera y Roberto Montero Granados, "La descentralización fiscal en América Latina. Balance de un proceso", Revista de Economía Institucional, vol. 17, n. ${ }^{\circ} 33,2015$, p. 14. Para el caso particular de Colombia, véase Jean Philippe Pening Gaviria, "Evaluación del proceso de descentralización en Colombia", Economía y desarrollo, vol. 2, n. ${ }^{\circ} 1,2003$, p. 125. Para una revisión crítica de los modelos de Administración pública y su implantación en América Latina, véase GuILLERMO FuENTES, María Cecilia Güemes y Carolina Isaza, "Modernizar y democratizar la Administración 
A pesar de lo anterior, hoy podemos decir que la descentralización está amenazada por la ineficiencia, la corrupción y el clientelismo que permea la política a nivel territorial ${ }^{4}$. Una característica transversal de estos problemas es el uso arbitrario de la administración y de sus medios para perseguir fines privados o de grupos particulares. No es un problema exclusivo de Colombia: Rodríguez-Arana lamenta, para el caso de España, que "la Administración fue tomada [...] por grupos políticos y a ella han servido casi en exclusiva. En lugar de explicar y justificar sus decisiones, se encerró en una torre de marfil, y decidió cerrarse a la sociedad consciente de que los partidos controlaban el resto de los poderes para acampar, más o menos en la impunidad"5.

A raíz de esta "crisis de la administración" -y paralelamente al desarrollo de conceptos como buena gobernanza ${ }^{6}$ - se ha ido decantando en algunas legislaciones del mundo la idea de que la Administración no debe ser un aparato cerrado, discrecional y arbitrario, restringido solo por unos requisitos legales generales ${ }^{7}$. A cambio, ha ido emergiendo el principio de la buena administración, que implica que las decisiones administrativas deben ser razonables, motivadas, además de consultadas e informadas a los ciudadanos involucrados ${ }^{8}$.

A pesar de lo anterior, son escasos los trabajos que analizan empíricamente cómo diferentes dimensiones de la buena administración pueden tener consecuencias reales sobre la calidad de la gestión pública y sus resultados. Por lo tanto, el objetivo de este trabajo es ilustrar, para el caso de los municipios de Colombia, la idea de que la buena administración promueve la eficiencia

Pública. Una mirada desde la realidad latinoamericana", Revista Enfoques: Ciencia Política y Administración Pública, vol. 7, n. ${ }^{\circ} 11,2009$.

4 Al respecto, véase ÓSCAR AlFONSO, "El oficio del corrupto y el detrimento al erario en el modelo territorial de Estado centralista-clientelista colombiano", en Juan Carlos Henao y Aníbal Rafael Zárate (eds.), Corrupción en Colombia, t. 4, Corrupción, Estado e instrumentos jurídicos, Bogotá: Universidad Externado de Colombia, 2018; David Ortiz Escobar y Luis Carlos CALIXTO, "Medición de la corrupción regional en Colombia: una propuesta de indicadores a partir de registros de organismos de investigación y control", en Juan Carlos Henao y Carolina Isaza (eds.), Corrupción en Colombia, t. 1, Corrupción, política y sociedad, Bogotá: Universidad Externado de Colombia, 2018.

5 Jaime Rodríguez Arana, "La buena administración como principio y como derecho fundamental en Europa", Revista de Derecho y Ciencias Sociales, 2014, p. 26.

6 Para una discusión del concepto y discurso de la "buena gobernanza", véase MARTIN Doornbos, "Good governance': The rise and decline of a policy metaphor?", Journal of Development Studies, vol. 37, n. ${ }^{\circ}$ 6, 2001, pp. 93-108; Bo RoTHSTEIN, The quality of government: Corruption, social trust, and inequality in international perspective, Chicago: University of Chicago Press, 2011.

7 Por ejemplo, el artículo 41 de la Carta Europea de Derechos Humanos, el principio de buon andamento consagrado en la Constitución italiana de 1947 y los artículos 31 y 103 de la Constitución española de 1978. Al respecto, véase Juli PonCe Solé, "Good Administration and Administrative Procedures", Indiana Journal of Global Legal Studies, 2005, p. 556.

8 Para un análisis de los elementos de la buena administración, véase JaIME RoDríGuEZ ArANA, óp. cit., p. 26. 
en las entidades territoriales. Concretamente, analizaremos los efectos de la transparencia -uno de los elementos constitutivos de una buena administración-sobre la eficiencia de los municipios como prestadores y productores de ciertos servicios.

Para cumplir con este objetivo, el trabajo se divide en tres partes: en la primera sección haremos una revisión de los conceptos de buena administración, transparencia y eficiencia de la gestión pública. Propondremos que la transparencia es un elemento constitutivo de la buena administración, mientras que la eficiencia debe ser entendida como el resultado de la buena administración en general, y de la transparencia en particular (1). En la segunda sección analizamos dos indicadores útiles para medir la transparencia y la eficiencia en los municipios de Colombia (2). En la tercera parte analizaremos empíricamente la relación entre transparencia -específicamente el cumplimiento de requisitos normativos relacionados con la información- y la eficiencia de la gestión municipal, entendida como una relación entre inputs y outputs (3).

\section{BUENA ADMINISTRACIÓN, TRANSPARENCIA Y EFICIENCIA DE LA GESTIÓN PÚBLICA}

El principio moderno de la buena administración es consecuencia del nuevo paradigma de la Administración pública, que se caracteriza por replantear la relación entre los ciudadanos y la Administración (1.1). Entre otros elementos que lo dotan de contenido, el principio de buena administración requiere que las decisiones administrativas sean motivadas y ejecutadas de manera abierta y transparente (1.2). Aunque la eficiencia de la Administración ha sido incluida como otro elemento definitorio de la buena administración, proponemos que esta debe ser vista como uno de sus principales resultados (1.3).

\subsection{EL PRINCIPIO MODERNO DE LA BUENA ADMINISTRACIÓN}

Según el paradigma tradicional, la relación entre los ciudadanos y la Administración pública es una relación jerárquica, en donde los "ciudadanos son vistos como subordinados de las autoridades públicas" ${ }^{\prime \prime}$, si bien sujetos de protección en el marco del Estado de derecho. La Administración es vista como una esfera de discrecionalidad en la que basta con acatar unas reglas generales que protegen a los ciudadanos de la arbitrariedad de los funcionarios. Por lo tanto, "el derecho administrativo tradicional no está especialmente interesado en buenas decisiones administrativas sino en la revisión judicial de las decisiones ilegales"10. Según este

9 Support for Improvement in Governance and Management, Good Administration through a Better System of Administrative Procedures, Kosovo: OCDE, 2012, pp. 1-49.

10 Juli Ponce Solé, "Good Administration and Administrative Procedures", óp. cit., p. 554. Bastardillas fuera del texto. 
paradigma, el respeto al Estado de derecho y la predictibilidad serían suficientes para que la administración sea calificada como "buena"11.

En contraste, los cambios ocurridos en lo que puede ser catalogado como el programa histórico del derecho administrativo ${ }^{12}$ llevan a una comprensión muy distinta de la Administración pública y de la relación entre esta y los ciudadanos, de maneara que se enriquece la lista de elementos que caracterizan a una buena administración ${ }^{13}$. En efecto, en el nuevo paradigma, "al ciudadano se le da un espacio como miembro activo, como un socio que puede contribuir al bienestar general. Sus inputs, cooperación y participación son alentados y buscados como una condición necesaria para la gobernanza democrática y eficiente, y para el desarrollo económico ${ }^{114}$. Lo anterior perfila el principio moderno de la buena administración, que va encaminado a dirigir o enmarcar la discrecionalidad administrativa de manera que las decisiones no solo sean legales, sino que también sean correctas ${ }^{15}$. El principio se basa en la idea de que "la actividad administrativa [...] no es solo una actividad sujeta a la ley, sino que, además, es una actividad que se expresa a través de buenas decisiones administrativas, debidamente motivadas, en términos contestables, como consecuencia de un previo procedimiento abierto y participativo" ${ }^{\prime 16}$.

Siguiendo a Bustamante Alarcón ${ }^{17}$, la buena administración es un concepto gemelo al del buen gobierno, aunque este último ha acaparado mayor atención en las discusiones sobre calidad de la gestión del ejecutivo. Para simplificar, el buen gobierno se refiere a la actuación de los gobernantes como dirigentes supremos del poder ejecutivo, mientras que la buena administración se refiere

11 "Not long ago, the main challenges for Good Administration were: i) respect for the rule of law and ii) predictability. Administrative decisions bad to be based on a valid legal provision circumscribing competence and setting its limits. In this way, decisions could be reviewed and controlled by the bierarchy and the judiciary. Predictability and accountability of administrative actions were ensured". SUPPORT FOR IMPROVEMENT in Governance and Management, óp. cit., p. 7.

12 Sobre este desarrollo histórico, ampliamente relacionado con los cambios sufridos por el modelo de Estado y los fines a su cargo, véase Jean-Bernard Auby, "Droit administratif et démocratie", in Régulation économique et démocratie, Paris: Dalloz, 2006, p. 17.

13 Por ejemplo, la Constitución española establece los elementos de acción efectiva, eficiencia, economía, coordinación y no arbitrariedad. Véase Juli PonCE Solé, "Good Administration and Administrative Procedures", óp. cit., p. 556.

14 Support For Improvement in Governance And Management, óp. cit.

15 La buena administración ha sido descrita como un principio de la Administración pública o como un derecho de los ciudadanos.

16 Pedro Spano Tardivo, "El principio de transparencia de la gestión pública en el marco de la teoría del bueno gobierno y la buena administración. La transformación de la Administración pública para la tutela de los derechos fundamentales a propósito de la provincia de Santa Fe", Revista Digital de la Asociación Argentina de Derecho Administrativo, 2016, pp. 225-268.

17 Reynaldo Bustamante Alarcón, Fundamentos políticos constitucionales del buen gobierno y la buena administración, Lima: Conferencia Estabilidad Jurídica y Fortalecimiento Institucional Público-Privado, 2015. 
a la actuación de la administración en su conjunto, es decir, incluye la gestión del aparato burocrático y de todos los funcionarios públicos ${ }^{18}$.

En su espíritu, la buena administración es una administración "que sirve objetivamente a la ciudadanía, que realiza su trabajo con racionalidad, justificando sus actuaciones y que se orienta continuamente al interés general"19. Si bien esta definición aún es muy abstracta, legislaciones y códigos de diferentes jurisdicciones han sugerido una lista de elementos que dotan de contenido a la buena administración y que la hacen un principio -o un derecho- practicable en el actuar de la Administración pública. Por ejemplo, la Carta Europea de los Derechos Fundamentales de 2000, y posteriormente de 2007, consagra el derecho a que las personas sean oídas antes de que la Administración tome decisiones que las afecte, el derecho a acceder a la información relevante en la toma de estas decisiones y la obligación de la Administración a motivar sus decisiones ${ }^{20-21}$. Además, la Carta consagra el derecho a la reparación por daños causados por las instituciones europeas y el derecho a comunicarse con las autoridades europeas en cualquiera de las lenguas oficiales de la Unión. Estos derechos de los ciudadanos europeos y obligaciones de las instituciones de la Unión fueron desarrollados en el Código Europeo de Buena Conducta de 2001.

A nivel iberoamericano, cabe señalar la Carta Iberoamericana de los Derechos y Deberes del Ciudadano en Relación con la Administración Pública de 2013, cuya finalidad es "el reconocimiento del derecho fundamental de la persona a la buena Administración pública y de sus derechos y deberes componentes $^{\prime \prime 22}$. Según la Carta, la actuación de la Administración pública debe estar regida por principios como la igualdad, la motivación adecuada, la eficacia, la eficiencia, la ética, la participación ciudadana y la publicidad.

Aunque el principio de buena administración no está consagrado explícitamente en la legislación colombiana, el artículo 209 de la Constitución Política indica que la "función administrativa está al servicio de los intereses generales y se desarrolla con fundamento en los principios de igualdad,

18 Para una reflexión sobre la diferencia entre buen gobierno y buena administración, véase José MeILÁN GIL, "El paradigma de la buena administración", Anuario da Facultade de Dereito da Universidade da Coruña, 2013, pp. 233-257.

19 Jaime Rodríguez Arana, óp. cit., p. 26.

20 Carta de los Derechos Fundamentales de la Unión Europea, Diario Oficial de las Comunidades Europeas, 2000, pp. 1-22.

21 Al respecto de la obligación de la motivación, Rodríguez Arana afirma que este derecho "es el más importante [...]. Llama la atención que este derecho se refiera a todas las resoluciones europeas sin excepción. Me parece un gran acierto la letra y el espíritu de este precepto. Sobre todo, porque una de las condiciones del ejercicio del poder en las democracias es que sea argumentado, razonado, motivado". JaIme Rodríguez Arana, óp. cit., p. 40.

22 Centro latinoamericano de Administración para el Desarrollo, Carta Iberoamericana de los Derechos y Deberes del Ciudadano en Relación con la Administración Pública, Panamá: XXIII Cumbre Iberoamericana de Jefes de Estado y de Gobierno, 2013, pp. 1-14. 
moralidad, eficacia, economía, celeridad, imparcialidad y publicidad". Varios de estos elementos coinciden con aquellos consagrados en los documentos de la Unión Europea y la Carta iberoamericana.

\subsection{Transparencia como elemento constitutivo DE LA BUENA ADMINISTRACIÓN}

Según Solé, "si bien transparencia y derecho a una buena administración tienen profundas relaciones, lo cierto es que son conceptos distintos ${ }^{\prime \prime 23}$. Aunque también es posible afirmar que la transparencia es un principio relevante para esferas diferentes a la buena administración (por ejemplo, para el buen gobierno), en aras de simplificar la exposición, coincidimos con Solé cuando argumenta que la transparencia es un instrumento de la buena administración. En efecto, la transparencia es uno de los elementos concretos que dota de contenido al principio de buena administración. De esta manera, una buena administración es, entre otras cosas, una administración transparente.

Por ejemplo, en la Carta Iberoamericana está consignado el principio de participación, según el cual los ciudadanos "podrán [...] estar presentes e influir en todas las cuestiones de interés general, (así como participar) en el control de la actividad administrativa"24. Así mismo, la Carta consagra el derecho de "publicidad y claridad de las normas, de los procedimientos y del entero del quehacer administrativo" ${ }^{\prime 25}$. Por último, el principio 22 habla de la "transparencia y acceso a la información de interés general" ${ }^{26}$. Lo anterior tiene sentido si se tiene en cuenta que, de acuerdo al nuevo paradigma de la gestión pública, una buena administración no puede ser una administración alejada del ciudadano. Cabe aclarar que la transparencia no es la simple revelación de la información concerniente a la ejecución y resultados de la Administración pública, sino la posibilidad de que los ciudadanos puedan activamente opinar e influir en el modo de actuación de la misma. De esta manera, el principio de transparencia, "supera aquel primer reconocimiento del derecho de acceso de la información pública y se abraza a la libre participación en la confección de políticas públicas y, en definitiva, a la libertad de expresión garantizada en la libre participación en la gestión de la cosa pública" ${ }^{\prime \prime 2}$. nacional del CLAD sobre la Reforma del Estado y de la Administración Pública, 2015, p. 1. Centro latinoamericano de Administración para el Desarrollo, óp. cit., p. 7.

25 Ibíd., p. 7

26 Ibíd., p. 9.

27 Pedro Spano Tardivo, óp. cit., pp. 225-268. 


\subsection{EFiciencia de la Administración pública como Resultado DE LA BUENA ADMINISTRACIÓN}

Aunque la eficiencia y la efectividad de la Administración han sido catalogados como características de la buena administración ${ }^{28}$, aquí propondremos que deben ser vistas como el resultado de la buena administración. En efecto, elementos de la buena administración como la transparencia, además de alinear a la administración con los intereses de los ciudadanos y protegerlos de la arbitrariedad, incentivan buenas prácticas para hacer un uso más eficiente de los recursos públicos. Según Hood, "el acceso abierto a la información y la eliminación del secreto se consideran una condición para la prevención de la corrupción y la promoción de la responsabilidad pública"29. En teoría, al exponer la gestión de las Administraciones y someterla al escrutinio de los ciudadanos, la transparencia desestimula la corrupción, la negligencia y el descuido en el uso de los recursos públicos.

Ahora bien, aunque existe evidencia que respalda la idea de que la calidad del gobierno y bajos niveles de corrupción mejoran la eficiencia del gobierno tanto a nivel nacional ${ }^{30} \mathrm{como}$ a nivel local ${ }^{31}$, la evidencia sobre la relación entre transparencia per se y eficiencia es mixta. Por ejemplo, Lepore y Pisano encontraron que la revelación de información en línea no mejora el desempeño de los gobiernos locales en Italia, aunque la atención de los medios sí lo hace ${ }^{32}$. Lo anterior motiva la pregunta de esta investigación: cla buena administración, específicamente la transparencia de la gestión pública, promueve la eficiencia de la gestión de los municipios de Colombia? Al respecto cabe señalar el estudio de Cano Muñoz ${ }^{33}$, quien encontró una correlación importante entre

28 "Good Administration is effective and efficient. Public authorities need to be successful in achieving the goals and bandling the public problems set for them by law and government, they need to use public resources in a way proportional to the results attained, they set clear objectives, evaluating past experience as well as the future impact of their action. Not imposing unnecessary burdens and costs on the citizens and business is another way to increase effectiveness and efficiency". SUPPORT FOR IMPROVEMENT IN GOVERNANCE AND MANAGEMENT, óp. cit., p. 9.

29 David Hood y David Heald, Transparency, London: Oxford University Press, 2001, pp. 700-704.

30 D. Hauner y A. Kyobe, "Determinants of government efficiency" World Development, vol. 38, n. ${ }^{\circ} 11,2010$, pp. 1527-1542.

31 Massimo Finocchiaro Castro, Calogero Guccio e Ilde Rizzo, "An assessment of the waste effects of corruption on infrastructure provision", International Tax and Public Finance, vol. 21, n. $^{\circ} 4,2014$, pp. 813-843

32 Luigi LePore y Sabrina PisAno, "Accountability and Performance of Italian Local Government Authorities: How Does e-Disclosure Affect Performance?", in Reshaping Accounting and Management Control Systems, Naples: Springer, 2017, pp. 277-288.

33 Luz Patricia Cano MuÑoz, La corrupción y la ineficiencia en el gasto público local y su impacto en la pobreza en Colombia, Bogotá: Fedesarrollo, 2013, pp. 1-30. 
los niveles de eficiencia y los niveles de transparencia para una muestra de municipios de Colombia ${ }^{34}$.

\section{MEDICIÓN DE LA TRANSPARENCIA Y DE LA EFICIENCIA EN LOS MUNICIPIOS DE COLOMBIA}

El análisis empírico de los conceptos analizados en la sección anterior requiere de un ejercicio de operacionalización y medición de los mismos. Este ejercicio es por naturaleza imperfecto y está bastante limitado por la información disponible. Para la medición de la transparencia de los gobiernos locales proponemos usar el Índice de Gobierno Abierto (IGA) realizado por la Procuraduría General de la Nación (2.1), mientras que para medir la eficiencia de los municipios tomamos la medición de eficiencia realizada por el DNP (2.2). Como se verá, a pesar de las dificultades metodológicas de estas mediciones ${ }^{35}$, es posible enlazar los conceptos de interés con dimensiones concretas de estos índices. Además, los dos conjuntos de datos están disponibles a nivel municipal, lo que hace más rico el análisis que cuando se usan solamente indicadores a nivel nacional o a nivel departamental.

\subsection{Medición de la transparencia: Índice de Gobierno Abierto (iga)}

El IGA parte del marco teórico de la cadena de valor de la gestión de información. La idea del índice es capturar, en un solo número (de 0 a 100), el nivel de cumplimiento normativo de los municipios en tres dimensiones: a) organización de la información, b) exposición de la información, y c) diálogo sobre la información. Subrayamos que el índice mide "el cumplimiento normativo", es decir, su apego a normas y procedimientos establecidos por la ley en materia de gestión y circulación de la información. En general, los diferentes componentes del IGA miden qué tanto las entidades reportan información de manera oportuna a los sistemas nacionales que recogen información de la gestión pública.

a) Organización de la información: Este rubro comprende, por una parte, el control interno que "procura que todas las actividades, operaciones y actuaciones, así como la administración de la información y los recursos, se realicen de acuerdo con las normas constitucionales y legales vigentes dentro de las políticas

34 Como se verá más adelante, el trabajo de Cano Muñoz toma la medida de transparencia de Transparencia por Colombia, que para el año del estudio comprendía 148 municipios de Colombia.

35 Los índices usados para medir la transparencia y la eficiencia de los municipios de Colombia pueden tener errores de medición debido, principalmente, a que surgen de registros alimentados por autoridades municipales y nacionales. Sin embargo, constituyen una buena aproximación para la medición de estos conceptos. 
trazadas por la dirección, y en atención a las metas u objetivos previstos ${ }^{1136}$. Por otro lado, la gestión documental "evalúa el nivel de implementación de la Ley 594 de 2000, mediante la cual se establecen las reglas y principios generales que regulan la función archivística en todas las entidades del Estado" ${ }^{37}$. Es posible afirmar que este último componente es un requisito para que la organización pueda ser transparente: sin una recolección y organización de información adecuada, esta no puede estar accesible a la ciudadanía.

b) Exposición de la información: "Incorpora actividades tendientes a disponer, difundir, presentar o reportar información, e implica una disposición abierta desde la entidad hacia actores externos como los organismos de seguimiento y control ${ }^{1 / 38}$. Cabe anotar que los mecanismos de reporte de información han tenido el objetivo de incrementar la transparencia bacia Bogotá, en el sentido en que estos insumos son usados por las entidades del gobierno central para evaluar y planear políticas públicas, así como hacer seguimiento a la gestión del gasto. Sin embargo, la idea implícita del gobierno abierto es que esta información también está disponible para los ciudadanos en el momento en que la requieran.

c) Diálogo de la información: "Se refiere a aquellos canales y acciones de socialización, explicación, justificación y retroalimentación con los grupos de interés en aplicación de los principios de democracia participativa y democratización de la gestión pública" ${ }^{\prime 39}$. Se trata de la otra dimensión de la transparencia: que los ciudadanos puedan interactuar con la administración a través de canales conocidos y expeditos, de tal manera que no sean solo receptores pasivos de la información.

Cabe aclarar que Transparencia por Colombia también ofrece una medida de transparencia para departamentos y ciudades capitales (ITEP ${ }^{40}$. La metodología de este indicador abarca otros aspectos diferentes al cumplimiento normativo. Sin embargo, el presente estudio se concentra en el IGA debido principalmente a que ofrece información para todos los municipios de Colombia.

La tabla 1 muestran algunas clasificaciones de los municipios de Colombia según el IGA.

36 Procuraduría General de la Nación, Índice de Gobierno Abierto (IGA). Resultados 2015. Análisis comparativo 2010-2015, Bogotá, 2016, pp. 1-128. Ibíd., p. 28.

38 Ibíd., p. 25 .

39 Ibíd., p. 25.

40 Transparencia por Colombia, Índice de transparencia departamental, gobernaciones y contralorías, Resultados 2015-2016, Colección de Documentos Observatorio de Integridad n. ${ }^{\circ}$ 18, Bogotá, marzo de 2017, Índice de transparencia municipal, Resultados 2015-2016, Colección de Documentos Observatorio de Integridad n. ${ }^{\circ}$ 17, Bogotá, marzo de 2017. 
Tabla 1. Algunas Clasificaciones de los municipios de Colombia SEGÚN EL IGA (2016)

\begin{tabular}{|c|c|c|c|c|c|c|}
\hline \multirow{2}{*}{ IGA } & \multicolumn{2}{|c|}{ Más de 100.000 habitantes } & \multicolumn{2}{|c|}{$\begin{array}{c}\text { Entre } 50.000 \text { y } 100.000 \\
\text { habitantes }\end{array}$} & \multicolumn{2}{|c|}{ Menos de 50.000 habitantes } \\
\hline & Municipio & Departamento & Municipio & Departamento & Municipio & Departamento \\
\hline \multirow{5}{*}{ Mejores } & Sogamoso & Boyacá & Mosquera & Cundinamarca & Chaparral & Tolima \\
\hline & Medellín & Antioquia & La Ceja & Antioquia & La Virginia & Risaralda \\
\hline & Bogotá D.C. & Cundinamarca & La Estrella & Antioquia & $\begin{array}{l}\text { Castilla la } \\
\text { Nueva }\end{array}$ & Meta \\
\hline & Itaguí & Antioquia & Acacias & Meta & Guarne & Antioquia \\
\hline & Villavicencio & Meta & Copacabana & Antioquia & Melgar & Tolima \\
\hline \multirow{5}{*}{ Peores } & Buenaventura & $\begin{array}{l}\text { Valle del } \\
\text { Cauca }\end{array}$ & $\begin{array}{l}\text { Valle del } \\
\text { Guamuez }\end{array}$ & Putumayo & Mosquera & Cundinamarca \\
\hline & Lorica & Córdoba & $\begin{array}{l}\text { Sabana } \\
\text { Larga }\end{array}$ & Bolívar & $\begin{array}{l}\text { Hatillo de } \\
\text { Loba }\end{array}$ & Bolívar \\
\hline & Tumaco & Nariño & Turbaco & Bolívar & $\begin{array}{l}\text { Barranco de } \\
\text { Loba }\end{array}$ & Bolívar \\
\hline & Jamundí & $\begin{array}{l}\text { Valle del } \\
\text { Cauca }\end{array}$ & Arjona & Bolívar & Santa Lucía & Atlántico \\
\hline & Magangué & Magdalena & San Onofre & Sucre & La Tola & Nariño \\
\hline
\end{tabular}

\subsection{Medición de la eficiencia del Departamento Nacional}

\section{de Planeación (DNP)}

El ejercicio realizado por el DNP pretende "medir la relación entre productos obtenidos e insumos utilizados por un municipio en el proceso de producción de bienes y de prestación de servicios básicos ${ }^{\prime \prime 41}$. La medición comprende tres grandes sectores: educación, salud y agua potable. A manera de ejemplo, uno de los productos del sector educativo es el número de alumnos matriculados. Los insumos utilizados por los municipios son el total de docentes oficiales, la inversión en educación diferente a gastos de nómina y el espacio disponible para aulas en establecimientos educativos oficiales. Un municipio es más eficiente cuanto puede producir más del bien en mención usando los mismos insumos.

El cálculo de la eficiencia se realiza usando la técnica de análisis envolvente de datos (DEA, por sus siglas en inglés), que no asume ninguna función de

41 Departamento Nacional de Planeación, Guía "Orientaciones para realizar la medición del desempeño integral municipal", Bogotá, 2017. 
producción particular. El resultado de la implementación de la metodología es un indicador de eficiencia (de 0 a 100) que permite realizar un escalafón de municipios. La tabla 2 muestran algunas clasificaciones de los municipios de Colombia según su nivel de eficiencia.

\section{Tabla 2. Algunas Clasificaciones de los municipios de Colombia SEGúN EL ÍNDICE DE EFICIENCIA DEL DNP (2016)}

\begin{tabular}{|c|c|c|c|c|c|c|}
\hline \multirow{2}{*}{ Eficiencia } & \multicolumn{2}{|c|}{ Más de 100.000 habitantes } & \multicolumn{2}{|c|}{$\begin{array}{c}\text { Entre } 50.000 \text { y } 100.000 \\
\text { habitantes }\end{array}$} & \multicolumn{2}{|c|}{ Menos de 50.000 habitantes } \\
\hline & Municipio & Departamento & Municipio & Departamento & Municipio & Departamento \\
\hline \multirow{5}{*}{ Mejores } & Bogotá D.C. & Cundinamarca & Ocaña & Santander & La Uribe & Meta \\
\hline & Medellín & Antioquia & $\begin{array}{l}\text { Villa del } \\
\text { Rosario }\end{array}$ & Santander & Villa Nueva & Guajira \\
\hline & Cali & $\begin{array}{l}\text { Valle del } \\
\text { Cauca }\end{array}$ & $\begin{array}{l}\text { Santa Rosa } \\
\text { de Cabal }\end{array}$ & Risaralda & Saravena & Arauca \\
\hline & Soledad & Atlántico & Chigorodó & Antioquia & Pasto & Nariño \\
\hline & Bucaramanga & Santander & Florida & Nariño & $\begin{array}{l}\text { La Jagua de } \\
\text { Ibirico }\end{array}$ & Cesar \\
\hline \multirow{5}{*}{ Peores } & Maicao & Guajira & $\begin{array}{l}\text { El Carmen de } \\
\text { Bolívar }\end{array}$ & Bolívar & Magui-Payan & Nariño \\
\hline & Lorica & Córdoba & Sahagún & Córdoba & Distracción & Guajira \\
\hline & Uribia & Guajira & Ayapel & Córdoba & Puerto Nariño & Amazonas \\
\hline & Manaure & Guajira & Orito & Putumayo & Dibulla & Guajira \\
\hline & Jamundí & $\begin{array}{l}\text { Valle del } \\
\text { Cauca }\end{array}$ & Puerto Asís & Putumayo & San Carlos & Córdoba \\
\hline
\end{tabular}

\section{RESULTADOS}

Las mediciones explicadas en la sección anterior son un insumo útil para explorar la relación entre transparencia y eficiencia en los municipios de Colombia. Más concretamente, cestá relacionado el cumplimiento normativo de los requisitos relativos al manejo de la información con la eficiencia de los gobiernos locales en la provisión de ciertos bienes públicos como la educación y la salud?

La gráfica 1 muestra una correlación positiva entre el índice de gobierno abierto y la eficiencia de los gobiernos municipales. Nótese que esta relación se cumple para diferentes segmentos de municipios, que hemos dividido según el número de habitantes. 


\section{GráfICA 1. IGA Y EFICIENCIA (2016)}

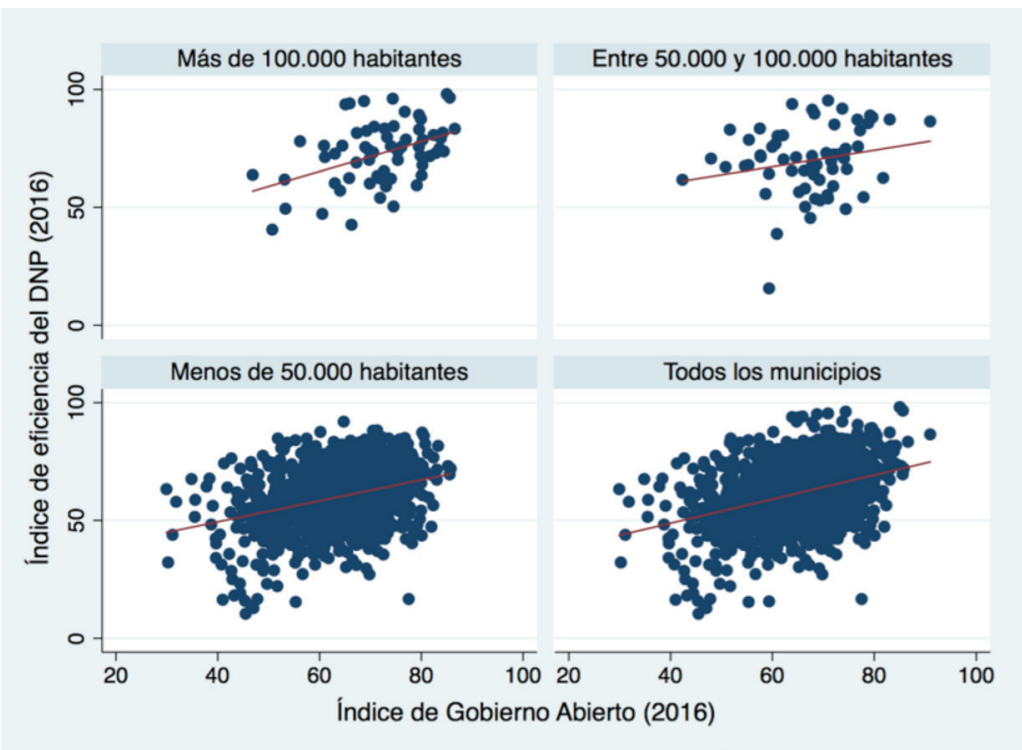

Para explorar mejor esta relación, estimamos un modelo de mínimos cuadrados ordinarios, en donde la variable explicada es la eficiencia de los municipios y la variable explicativa es el IGA (estimación 1). Con el fin de corregir posibles sesgos del estimador del coeficiente, añadimos una serie de controles relacionados con la población del municipio (DANE) ${ }^{42}$ y su presupuesto $(\mathrm{DNP})^{43}$ (estimación 2). Finalmente, añadimos una variable proxy de control relacionada con el nivel de educación de los habitantes del municipio: la tasa de alfabetización en 2005 (DANE) ${ }^{44}$ (estimación 3). Lo anterior, para abordar una de las fuentes de endogeneidad del modelo: municipios más educados podrían tener una Administración más eficiente y al mismo tiempo ser más transparentes debido a que los ciudadanos demandan más información de la administración local ${ }^{45}$ (tabla 3 ).

42 Los datos asociados a la población son estimaciones hechas por el Departamento Administrativo Nacional de Estadística para el año 2016

43 Cálculos de los ingresos por concepto de impuestos y transferencias hechas por el Departamento Nacional de Planeación para el año 2016

44 Datos calculados por el Departamento Administrativo Nacional de Estadística para el año 2005.

45 Al respecto, Benito, Bastida y García encontraron una relación significativa entre los indicadores de eficiencia y los niveles de educación para los municipios españoles. BERNARdino Benito, Francisco Bastida y José García, "Explaining differences in efficiency: an application to Spanish municipalities", Journal of Applied Economics, 2010, pp. 515-528. 
Tabla 3. Resultados de las estimaciones (modelo 1). Variable explicada: EFICIENCIA DE LOS GOBIERNOS MUNICIPALES SEGÚN EL DNP (2016)

\begin{tabular}{|l|c|c|c|}
\hline & $(1)$ & $(2)$ & $(3)$ \\
\hline Total IGA & $0,511^{* * *}$ & $0,472^{* * *}$ & $0,342^{* * *}$ \\
\hline & $(0,0455)$ & $(0,0452)$ & $(0,0460)$ \\
\hline Población en miles & & $0,0661^{* * *}$ & $0,0579^{* * *}$ \\
\hline Presupuesto en millones & & $(0,0181)$ & $(0,0165)$ \\
\hline & & $-36,02^{* * *}$ & $-31,52^{* * *}$ \\
\hline Tasa de alfabetización en 2005 & & $(10,57)$ & $(9,645)$ \\
\hline & & & $0,4466^{* * *}$ \\
\hline Observaciones & & & $(0,0566)$ \\
\hline
\end{tabular}

Notas:

Errores estándar entre paréntesis.

$* p<0,10$

** $p<0,05$

$* * * \mathrm{p}<0,01$

La tercera estimación indica que, controlando por la tasa de alfabetización en 2005, la población y el presupuesto del municipio, aumentar un punto (de 0 a 100) en el IGA está asociado a un aumento promedio de 0,34 puntos (de 0 a 100) en el índice de eficiencia del DNP. Esto constituye una evidencia preliminar de que la transparencia de los gobiernos municipales sí tiene un efecto sobre la eficiencia de las Administraciones. Es interesante señalar además que el tamaño de la población tiene un efecto positivo, pequeño, pero estadísticamente significativo sobre el nivel de eficiencia, mientras que el presupuesto tiene un efecto negativo. Por otro lado, no sorprende que la tasa de alfabetización en 2005 está positivamente correlacionada con la eficiencia: municipios históricamente más educados tienen a ser más eficientes.

El IGA es un indicador comprensivo de la gestión y la apertura de la información por parte de las entidades territoriales, pero algunos de sus componentes están más estrechamente ligados al concepto de transparencia hacia las organizaciones del Estado y hacia la ciudadanía (ver anexo).

- Gestión documental: "El indicador Ley de Archivos define el resultado de esta categoría, a través del cual se evalúa el nivel de implementación de la Ley 594 de 2000, mediante la cual se establecen las reglas y principios generales que regulan la función archivística en todas las entidades del Estado" ${ }^{\prime \prime}$. 
- Exposición de la información: "El componente Exposición de la Información incorpora actividades tendientes a disponer, difundir, presentar o reportar información, e implica una disposición abierta desde la entidad hacia actores externos como los organismos de seguimiento y control y la ciudadanía"47. Está más asociado con transparencia a hacia los entes del Estado, ya que son reportes que se le presentan directamente a los organismos del Estado como el Ministerio de Hacienda, DNP, etc.

- Transparencia y gobierno electrónico: Mide los "avances para aprovechar los avances en materia de conectividad para acercar al ciudadano a las instituciones, facilitar el acceso a la información, agilizar los trámites y hacer más transparente el ejercicio de la función pública" 48.

- Rendición de cuentas: "A través del indicador Audiencias Públicas se evalúan los mecanismos utilizados por las autoridades territoriales para facilitar que la ciudadanía y las organizaciones de la sociedad civil se involucren en la formulación, ejecución, control y evaluación de la gestión pública, y para rendir cuentas sobre la gestión ejecutada"49.

- Atención al ciudadano: "Esta categoría agrupa dos de los instrumentos que permiten evaluar si las entidades están brindando o no un servicio a la ciudadanía de manera oportuna y de calidad"50.

Para analizar específicamente cuáles de los componentes del IGA relacionados con la transparencia están asociados a la eficiencia de los gobiernos municipales, estimamos un nuevo modelo en donde las variables explicativas son los componentes del IGA anteriormente descritos. Los resultados se muestran en la tabla 4.

Tabla 4. Resultados de las estimaciones (modelo 2). Variable explicada: EFICIENCIA DE LOS GOBIERNOS MUNICIPALES SEGÚN EL DNP (2016)

\begin{tabular}{|l|c|c|c|}
\hline & $(1)$ & $(2)$ & $(3)$ \\
\hline Gestión documental & $0,0708^{* * *}$ & $0,0746^{* * * *}$ & $0,0570^{* * * *}$ \\
\hline Exposición de la información & $(0,0154)$ & $(0,0146)$ & $(0,0142)$ \\
\hline & $0,212^{* * * *}$ & $0,205^{* * * *}$ & $0,144^{* * * *}$ \\
\hline Transparencia y gobierno electrónico & $(0,0402)$ & $(0,0391)$ & $(0,0372)$ \\
\hline & $0,0967^{* * * *}$ & $0,0743^{* * *}$ & $0,0502^{*}$ \\
\hline & $(0,0274)$ & $(0,0269)$ & $(0,0259)$ \\
\hline
\end{tabular}

47 Ibíd

48 Ibíd., p. 36.

49 Ibíd., p. 37.

50 Ibíd., p. 38. 


\begin{tabular}{|l|c|c|c|}
\hline & $(1)$ & $(2)$ & $(3)$ \\
\hline Rendición de cuentas & 0,0318 & 0,0328 & 0,0315 \\
\hline Atención al ciudadano & $(0,0253)$ & $(0,0246)$ & $(0,0227)$ \\
\hline & 0,0431 & 0,0361 & 0,0207 \\
\hline Población en miles & $(0,0362)$ & $(0,0358)$ & $(0,0344)$ \\
\hline & & $0,0691^{* * *}$ & $0,0603^{* * * *}$ \\
\hline Presupuesto en millones & & $(0,0180)$ & $(0,0164)$ \\
\hline & & $-37,77^{* * * *}$ & $-32,89^{* * * *}$ \\
\hline Tasa de alfabetización en 2005 & & $(10,53)$ & $(9,588)$ \\
\hline & & & $0,447^{* * *}$ \\
\hline Observación & & & $(0,0565)$ \\
\hline
\end{tabular}

Notas:

Errores estándares robustos en paréntesis.

$* \mathrm{p}<0,10$

$* * \mathrm{p}<0,05$

$* * * \mathrm{p}<0,01$

Las estimaciones arrojan que solo los coeficientes de gestión documental, exposición de la información y transparencia y gobierno electrónico son estadísticamente significativos. El coeficiente con mayor magnitud es el de exposición de la información. Controlando por los otros componentes, por la población y el presupuesto, así como por la tasa de alfabetización en 2005, un aumento de un punto en este componente (de 0 a 100) está asociado a un aumento promedio de 0,14 en el indicador de eficiencia.

Estos resultados concuerdan con los hallazgos de Cano Muñoz, quien también encontró una relación positiva entre la transparencia y la eficiencia de los gobiernos municipales. Según el estudio de Cano Muñoz, un aumento de una unidad en el índice de transparencia aumenta el indicador de eficiencia en $0,15^{[51]}$, magnitud similar al coeficiente estimado del componente de exposición de la información del IGA.

51 Este resultado es el efecto del aumento de la transparencia sobre la eficiencia promedio de los servicios de educación y salud para una muestra de 148 municipios colombianos. En este estudio se calcula la eficiencia usando la metodología DEA y se toma la medida de transparencia de Transparencia por Colombia. Ver CANO MuÑoz, óp. cit. 


\section{CONCLUSIONES}

En este artículo exploramos la relación entre el moderno concepto de buena administración, la transparencia y la eficiencia. Las normas y la doctrina analizadas concuerdan en que la transparencia es uno de los elementos constitutivos de la buena administración, pues esta supone que los ciudadanos estén debidamente informados de las actuaciones administrativas y de las motivaciones de estas actuaciones, y que además tengan acceso a canales para comunicarse con la administración. Proponemos, además, que la eficiencia no debe ser incluida mecánicamente como uno de los elementos que constituyen el principio de la buena administración, sino que debe ser entendida como su resultado, como uno de los fines que debe perseguir el gobierno en general y la administración en particular.

Con miras a analizar empíricamente la relación entre estos conceptos, tomamos las únicas medidas de transparencia y eficiencia que existen para todos los municipios de Colombia en 2016: el IGA y el índice de eficiencia del DNP. Cabe anotar que estas no reflejan exactamente los conceptos abstractos de transparencia, eficiencia o buena administración, pero son los únicos insumos existentes para abordar empíricamente estos conceptos. Contar con medidas más robustas de transparencia y eficiencia a nivel municipal facilitaría enormemente cualquier investigación que pretenda abordar la relación entre estas variables de manera cuantitativa.

Los resultados de los ejercicios empíricos mostraron que la transparencia -estrechamente entendida a través de los criterios de cumplimiento normativo que mide el IGA- sí tiene una asociación estadísticamente significativa con la eficiencia de los gobiernos municipales. Esta relación persiste incluso controlando por características como la población de los municipios, su presupuesto y el proxy de nivel educativo (la tasa de alfabetización de 2005). Según las estimaciones, un aumento de un punto en la transparencia está asociado a un aumento de entre 0,14 y 0,34 puntos en la eficiencia, según el proxy de transparencia utilizado. Futuras investigaciones podrían implementar estrategias más sofisticadas (a través de un panel de datos o variables instrumentales) con el fin de decantar conclusiones causales sobre el efecto de la buena administración en general, y de la transparencia en particular, sobre la eficiencia de las Administraciones. 


\section{BIBLIOGRAFÍA}

Auby, Jean-Bernard. "Droit administratif et démocratie", in Régulation économique et démocratie, Paris: Dalloz, 2006.

Alfonso, Óscar. "El oficio del corrupto y el detrimento al erario en el modelo territorial de Estado centralista-clientelista colombiano", en Juan Carlos Henao y Aníbal Rafael Zárate (eds.), Corrupción en Colombia, t. 4 Corrupción, Estado e instrumentos jurídicos. Bogotá: Universidad Externado de Colombia, 2018.

Benito, Bernardino, Francisco Bastida y José García. "Explaining differences in efficiency: an application to Spanish municipalities", Journal of Applied Economics, 2010.

Bustamante Alarcón, Reynaldo. Fundamentos políticos constitucionales del buen gobierno y la buena administración, Lima: Conferencia Estabilidad Jurídica y Fortalecimiento Institucional Público-Privado, 2015.

Cano Muñoz, Luz Patricia. La corrupción y la ineficiencia en el gasto público local y su impacto en la pobreza en Colombia, Bogotá: Fededesarrollo, 2013.

Carta de los Derechos Fundamentales de la Unión Europea, Diario Oficial de las Comunidades Europeas, 2000.

Centro latinoamericano de Administración para el Desarrollo. Carta Iberoamericana de los Derechos y Deberes del Ciudadano en Relación con la Administración Pública, Panamá: XxiII Cumbre Iberoamericana de Jefes de Estado y de Gobierno, 2013.

Departamento Nacional de Planeación. Guía "Orientaciones para realizar la medición del desempeño integral municipal", Bogotá, 2017.

DoOrnbos, MARTIN. "Good governance': The rise and decline of a policy metaphor?", Journal of Development Studies, vol. 37, n. ${ }^{\circ}$ 6, 2001.

Falleti, Tulia G. Decentralization and subnational politics in Latin America. Cambridge: Cambridge University Press, 2010.

Finocchiaro Castro, Massimo, Calogero Guccio e Ilde Rizzo. "An assessment of the waste effects of corruption on infrastructure provision", International Tax and Public Finance, vol. 21, n. ${ }^{\circ}$ 4, 2014.

Fuentes, Guillermo, María Cecilia Güemes y Carolina Isaza. "Modernizar y democratizar la Administración Pública. Una mirada desde la realidad latinoamericana", Revista Enfoques: Ciencia Política y Administración Pública, vol. 7, n. ${ }^{\circ}$ 11, 2009.

Hauner, D. y A. Kyobe. "Determinants of government efficiency", World Development, vol. $38, n^{\circ} .11,2010$. 
Hood, Christopher y David Heald. Transparency, London: Oxford University Press, 2001.

Lepore, Luigi y Sabrina Pisano. "Accountability and Performance of Italian Local Government Authorities: How Does e-Disclosure Affect Performance?", in Reshaping Accounting and Management Control Systems, Naples: Springer, 2017.

MeILÁn GiL, José. "El paradigma de la buena administración", Anuario da Facultade de Dereito da Universidade da Coruña, 2013

Ortiz Escobar, David y Luis Carlos Calixto. "Medición de la corrupción regional en Colombia: una propuesta de indicadores a partir de registros de organismos de investigación y control", en Juan Carlos Henao y Carolina Isaza (eds.), Corrupción en Colombia, t. 1, Corrupción, política y sociedad, Bogotá: Universidad Externado de Colombia, 2018.

Pening Gaviria, Jean Philippe. "Evaluación del proceso de descentralización en Colombia", Economía y desarrollo, vol. 2, n. ${ }^{\circ}$ 1, 2003.

Pinilla-Rodríguez, Diego E., Juan de Dios Jiménez Aguilera y Roberto Montero Granados. "La descentralización fiscal en América Latina. Balance de un proceso", Revista de Economía Institucional, vol. 17, n. ${ }^{\circ} 33,2015$.

Ponce Solé, Juli. "Good Administration and Administrative Procedures", Indiana Journal of Global Legal Studies, 2005.

POnCE Solé, Juli. Transparencia y derecho a una buena administración, Lima: XX Congreso Internacional del CLAD sobre la Reforma del Estado y de la Administración Pública, 2015.

Procuraduría General de la Nación. Índice de Gobierno Abierto (IGA). Resultados 2015 - Análisis comparativo 2010-2015, Bogotá, 2016.

Rodríguez Arana, Jaime. "La buena administración como principio y como derecho fundamental en Europa", Revista de Derecho y Ciencias Sociales, 2014.

RoTHSTEIN, Bo. The quality of government: Corruption, social trust, and inequality in international perspective. Chicago: University of Chicago Press, 2011.

Spano Tardivo, Pedro. "El principio de transparencia de la gestión pública en el marco de la teoría del bueno gobierno y la buena administración. La transformación de la Administración Pública para la tutela de los derechos fundamentales a propósito de la provincia de Santa Fe", Revista Digital de la Asociación Argentina de Derecho Administrativo, 2016.

Support For Improvement in Governance and Management. Good Administration through a Better System of Administrative Procedures, Kosovo: OCDE, 2012. 
TransPaRenCia POR Colombia. Índice de transparencia departamental, gobernaciones y contralorías, Resultados 2015-2016, Colección de Documentos Observatorio de Integridad n. ${ }^{\circ}$ 18, Bogotá, marzo de 2017.

Transparencia pOr Colombia. Índice de transparencia municipal, Resultados 2015 2016, Colección de Documentos Observatorio de Integridad n. ${ }^{\circ}$ 17, Bogotá, marzo de 2017. 


\section{ANEXO}

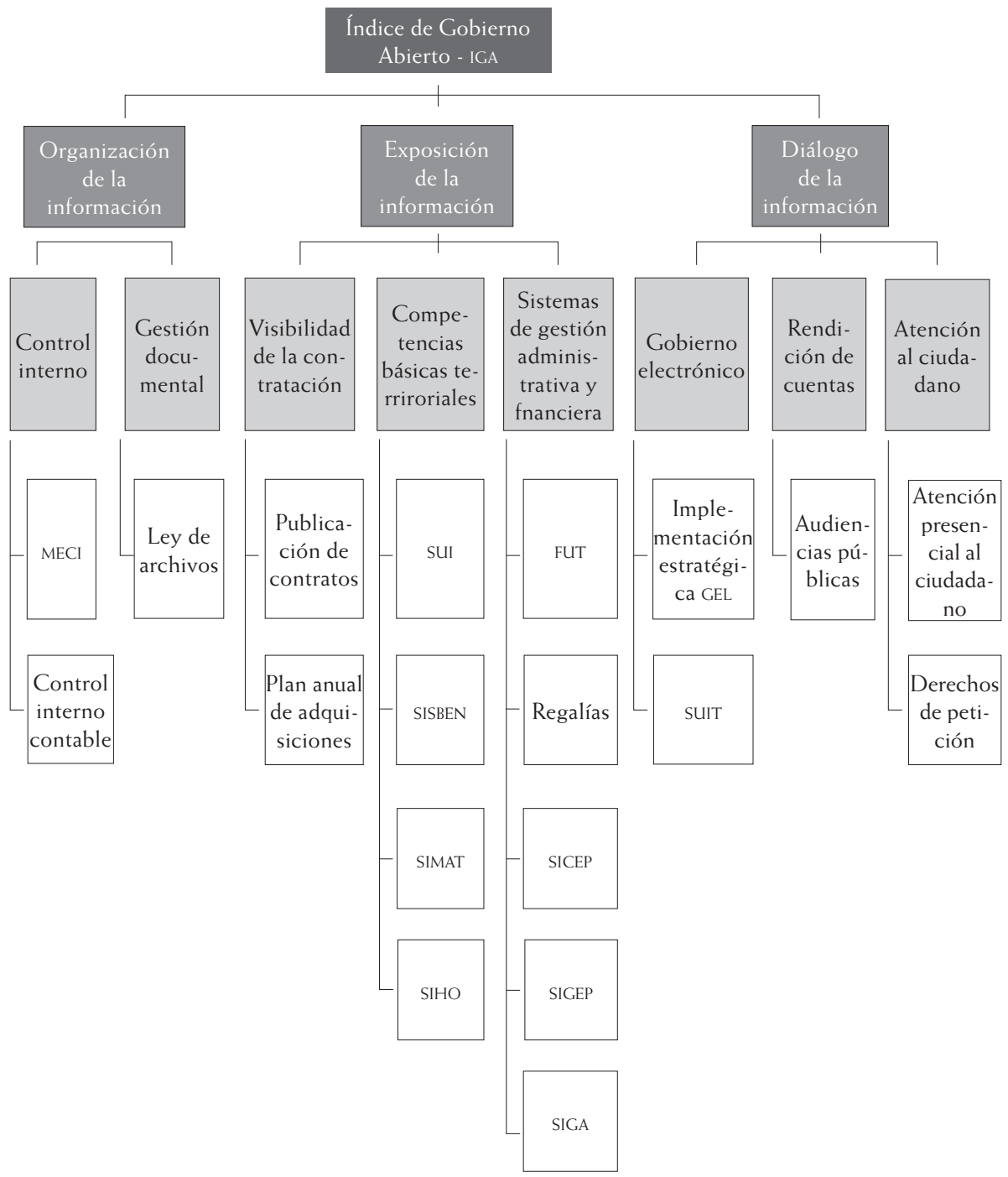

Fuente: Procuraduría General de la Nación. Índice de Gobierno Abierto (IGA). Resultados 2015 - Análisis comparativo 2010-2015, Bogotá, 2016, p. 42 